Provided by the author(s) and University of Galway in accordance with publisher policies. Please cite the published version when available.

\begin{tabular}{|c|l|}
\hline Title & $\begin{array}{l}\text { Comparing temporal event-coding in patients with first episode } \\
\text { psychosis and chronic schizophrenia }\end{array}$ \\
\hline Author(s) & $\begin{array}{l}\text { Schmidt, Heike; McFarland, John; McDonald, Colm; Elliott, } \\
\text { Mark }\end{array}$ \\
\hline $\begin{array}{c}\text { Publication } \\
\text { Date }\end{array}$ & 2008-06 \\
\hline $\begin{array}{c}\text { Publication } \\
\text { Information }\end{array}$ & $\begin{array}{l}\text { Schmidt, H., McFarland, J., McDonald, C., \& Elliott, M. A. } \\
\text { episode psychosis and chronic schizophrenia. Schizophrenia } \\
\text { Research, 102(1-3, Supplement 2), 107-108. }\end{array}$ \\
\hline $\begin{array}{c}\text { Publisher } \\
\text { Elsevier }\end{array}$ \\
\hline $\begin{array}{c}\text { Link to } \\
\text { publisher's } \\
\text { version }\end{array}$ & http://dx.doi.org/10.1016/S0920-9964(08)70324-4 \\
\hline $\begin{array}{c}\text { Item record } \\
\text { http://hdl.handle.net/10379/1505 }\end{array}$ \\
\hline
\end{tabular}

Downloaded 2023-04-26T09:51:13Z

Some rights reserved. For more information, please see the item record link above. 


\title{
COMPARING TEMPORAL EVENT-CODING IN PATIENTS WITH FIRST- EPISODE PSYCHOSIS AND CHRONIC SCHIZOPHRENIA
}

\author{
Heike Schmidt ${ }^{\mathrm{a}, \mathrm{b}}$, John McFarland ${ }^{\mathrm{b}}$, Anne Giersch ${ }^{\mathrm{c}}$, Colm McDonald ${ }^{\mathrm{b}}$ and Mark A. Elliott ${ }^{\mathrm{a}}$ \\ ${ }^{a}$ Department of Psychology, National University of Ireland, Galway, Republic of Ireland \\ ${ }^{b}$ Department of Psychiatry, University College Hospital Galway, Republic of Ireland \\ ${ }^{c}$ INSERM U666, Centre Hospitalier Régional de Strasbourg, Pôle de Psychiatrie, BP 406, \\ 67091 Strasbour Cedex, France
}

\begin{abstract}
Cognitive impairments are a core feature of schizophrenia. It has been suggested that the underlying cause of the cognitive deficits in schizophrenia is an impaired ability to sequence mental activity in time. Various studies have found increased time windows where patients with schizophrenia judge stimuli to appear simultaneously. However, very little is known if these deficits are already present in first-episode psychosis (FEP) or if they develop gradually over time. This study compared the subjective evaluation of temporal structure between healthy controls, chronic schizophrenic and FEP patients using an experimental approach involving judgments of simultaneity of visually presented stimuli. The results suggest that patients required longer delays between stimuli to detect asynchrony, although FEP patients exhibit shorter windows of simultaneity compared to the chronic sample. These data indicate that FEP patients do not appear to have the same impairments in event-coding in time as do chronic patients but show substantial variability.
\end{abstract}

Andreasen (1999, p.784) has suggested that schizophrenia is defined in terms of cognitive dysmetria, described as "a disruption of the fluid, coordinated sequences of thought and action that are the hallmark of normal cognition". This theory could explain how difficulties with regard to time phenomenology are related to the cognitive impairments typically observed in chronic schizophrenia. It has been suggested that these deficits may be due to disturbed functioning of the basal ganglia and the fronto-thalamic networks, which could also explain the deficits in other cognitive functions such as attention and memory.

Timing is crucial for most cognitive activities and having such impairments has wide ranging consequences. Various studies have shown that people with schizophrenia have problems estimating the duration of events (Davalos et al., 2002) or whether two stimuli appear simultaneously or not (Elliott et al., 2006, Giersch et al., 2008). These results suggest that patients with schizophrenia experience an abnormally large window of time during which two events will be judged to occur simultaneously (Giersch et al., 2008). This could be indicative of an underlying problem in functional coordination at a brain systems level and thereby many of the psycho-cognitive disorders found in schizophrenia. Most research on time perception and estimation has focused on chronic schizophrenia but very little is known if these abnormalities are also present at the onset of illness, i.e. in first-episode psychosis (FEP). Due to their minimal exposure to medication, FEP patients could provide more insight into the nature of the timing abnormalities typically observed in chronic schizophrenic patients.

The aim of the present study was to investigate the nature of time perception deficits observed in chronic schizophrenic patients and to compare them with FEP patients and healthy controls. 


\section{Method}

\section{Participants}

21 FEP patients (13 males, 6 females, mean age: 25.0 years), 20 chronic schizophrenic patients (14 males, 6 females, mean age: 32.2 years) and 16 matched healthy controls (10 males, 6 females, mean age: 22.8 years) participated in this study. The FEP sample comprised 4 schizophrenic patients, 5 with schizophreniform disorder, 5 with bipolar disorder with psychotic features, 3 not-otherwise specified psychosis patients, 1 substance induced psychosis and 1 with depressive disorder with psychotic features. All but 5 of the chronic sample were on clozapine and all but one (who was medication free) of the FEP sample were receiving atypical neuroleptic treatment for less than 6 weeks.

\section{Procedure:}

The present study used two experiments using the method of limits to determine visual simultaneity thresholds.

Experiment 1:

\section{Apparatus and Stimuli:}

Stimuli were presented on a Pentium 4 PC running Windows XP equipped with a Cambridge Research Systems (Rochester, Kent, UK) visual stimulus generator (ViSaGe), which was programmed in C language using the VSG software library. The visual stimuli were presented using a Mitsubishi Diamond Pro 2070SB monitor with the refresh rate set to $120 \mathrm{~Hz}$.

The target stimuli consisted of two vertical gray bars separated by $13^{\circ}$ of visual angle at a viewing distance of $100 \mathrm{~cm}$ at which each bar subtended $3^{\circ} \times 10^{\circ}$ of visual angle. Target bars increased luminance twice: The first increase (ranging from a background of $0.06 \mathrm{~cd} / \mathrm{m}^{2}$ to a peak luminance of $14.4 \mathrm{~cd} / \mathrm{m}^{2}$ ) was masked to make it below detection thresholds, while the second increase (ranging from $14.4 \mathrm{~cd} / \mathrm{m}^{2}$ to $29.8 \mathrm{~cd} / \mathrm{m}^{2}$ ) occurred in the absence of masking flankers. It was to the second increase that observers made their judgment of the simultaneity or asynchrony of the luminance increases between bars. Stimulus presentation occurred in an environment of low intensity, ambient light $\left(0.1 \mathrm{~cd} / \mathrm{m}^{2}\right)$ to reduce the impact of onscreen persistence.

The mask took the form of 6 flanker bars of identical dimensions to the target bars but the flankers were oriented pseudo-randomly $45^{\circ}$ to the left or right of the vertical meridian. The masking bars onset in pseudo-random order and temporally interleaved with the first change in target-bar luminance. The first change in target bar luminance occurred in two conditions; subthreshold synchrony $\left(\mathrm{SB}_{\mathrm{S}}\right)$ or asynchrony $\left(\mathrm{SB}_{\mathrm{A}}\right)$. In $\mathrm{SB}_{\mathrm{S}}$ the two bars started to change luminance at the same time. In $\mathrm{SB}_{\mathrm{A}}$, the two bars proceeded to change luminance at SOAs no lower than a previously determined simultaneity threshold for two bars presented in isolation (referred to as first staircase procedure) and no higher than a previously determined simultaneity threshold in the presence of flankers (second staircase procedure). This way, targets could change luminance at intervals which did not descend below the intervals at which targets would ordinarily be perceived as simultaneous (without flankers) and at the same time maintained at an upper level which was still below detection threshold (with flankers).

Procedure: This experiment consisted of three parts, two staircase procedures to determine the lower and upper simultaneity thresholds and the main experiment.

Staircase Procedures: Two staircase procedures were run to determine lower and upper simultaneity thresholds. In the lower threshold procedure, flanking bars were absent. In the second staircase procedure, flankers accompanied the luminance changes in order to obtain the upper threshold). These were used to place the prime asynchrony (derived from the 
median value between lower and upper thresholds) below detection in the main experiment. Participants responded to whether they found the second luminance change to be synchronous or asynchronous. Simultaneity thresholds were measured in terms of the delay between the onset of the first and the second bar, i.e. bar-bar stimulus onset asynchrony (SOA). A stochastic approximation procedure (Treutwein, 1995) was used to determine lower and upper simultaneity thresholds. This means that the 2 bars were initially presented with an SOA above threshold and then gradually being reduced on a trial-by-trial basis until the subject responds 'synchronous'. An initial threshold of $80 \mathrm{~ms}$ began the adaptive procedure. Both lower and upper threshold were determined seperately and at least twice in order to ensure stable tresholds as these were needed for the main experiment. In both staircase procedures, stimulus presentations were preceded by a $500 \mathrm{~ms}$ presentation of a fixation frame (four corner junctions), comprising a $13^{\circ} \times 13^{\circ}$ square region, within which the stimuli were presented.

Main experiment: Upon presentation of synchronous or asynchronous flanker luminance changes, experimental targets increased luminance within the flanker sequence, and again $150 \mathrm{~ms}$ after the flanker display had terminated. The first of these target SOAs was set at $0 \mathrm{~ms}$ for a synchronous prime, or within the range of SOAs circumscribed by the lower and upper threshold for an asynchronous prime. In contrast to the procedure used to determine the upper threshold, a second change in target luminance occured at the location of the premask bars and participants had to report whether this change in luminance was synchronous or asynchronous. There were 12 target SOAs (each level presented 40 times) while subthreshold SOAs were pseudo-randomly varied between the lower and upper thresholds established by the staircase procedures. The targets retained their luminance level for 2 seconds before the next trial commenced, and observers reported whether they perceived luminance-change synchrony or asynchrony of the targets. The main experiment was conducted in one session of 10 blocks comprising 48 trials per block and participants were advised to take breaks in between. In all cases, participants used the keyboard to respond by pressing the letters ' $F$ ' for synchronous and ' $J$ ' for asynchronous bars. The space bar was pressed to start a trial block.

\section{Experiment 2:}

The perception of simultaneity of simple visual stimuli was measured using a twoalternative forced-choice task run in Matlab ${ }^{\mathrm{TM}}$ (version 7.0) on a DELL computer (Windows 98) with a 15 inch monitor (refresh rate set to $120 \mathrm{~Hz}$ ). The participant's task was to determine whether they perceived the visual stimuli as 'simultaneous', that is presented together, or as 'asynchronous', i.e. presented in rapid succession. The stimuli consisted of two vertical grey bars, separated by $13^{\circ}$ of visual angle at a viewing distance of $100 \mathrm{~cm}$ at which each bar subtended $3^{\circ} \times 10^{\circ}$ of visual angle. The bars were displayed on a black background in an environment of low intensity ambient light $(0.1 \mathrm{~cd} / \mathrm{m} 2)$. The bars flickered on first presentation before judgement of the simultaneity was made. The stimuli also increased and decreased in subthreshold luminance (peak luminance of $14.4 \mathrm{~cd} / \mathrm{m}^{2}$ ) throughout the entire procedure. Participants made their response after each trial presentation by pressing the response keys "q" if the bars were perceived to be simultaneous and "p" for asynchronous bars. This paradigm incorporated an adaptive staircase technique whereby synchronies were increased or decreased in a stepwise fashion until judgements of simultaneity changed. This procedure was repeated until fifteen estimates of the threshold were obtained. The trials were separated by a 2 second inter-trial interval. 


\section{Results and Discussion}

\section{Experiment 1:}

Psychometric functions (PFs) were calculated individually. A preliminary inspection of the data revealed a high guess rate, which recommended correction. On this basis, the individual data were submitted to the following probability-based correction:

$$
P_{\text {adj }}(x)=\frac{P(x)}{P(0)}
$$

where $\mathrm{P}(0)$ is percentage of 'synchronous response' for 'subthreshold simultaneity (i.e. a subthreshold $\mathrm{SOA}=0$ ). This was done to eliminate the problem of a bias towards asynchronous responses. The individual thresholds were calculated as the SOA corresponding to a rate of $50 \%$ simultaneity responses giving an intermediate point between simultaneous and asynchronous responses. It was not possible to derive psychometric functions for the majority of chronic patients, hence the analysis was only carried out on 6 chronic patients, 8 FEP patients and 12 healthy controls.

A one-way ANOVA revealed significant differences in the overall thresholds between chronic, FEP patients and healthy controls $\left(\mathrm{F}_{2,23}=6.0, \mathrm{p}<0.05\right)$. Post-hoc tests showed that FEP patients (50 milliseconds) and healthy controls (47 milliseconds) had much lower simultaneity thresholds as compared to the chronic sample (79 milliseconds). The premask had a significant effect on thresholds $\left(\mathrm{F}_{1,22}=12.8\right.$, $\left.\mathrm{p}<0.01\right)$ with thresholds were significantly higher when the premask bars changed luminance simultaneously (FEP patients: 52 milliseconds, Chronic patients: 84 milliseconds, controls: 51 milliseconds) as compared to asynchronously (FEP patients: 47 milliseconds, chronic patients: 74 milliseconds, controls: 44 milliseconds). As can be seen in figure 1, particularly the chronic and FEP group showed large variability in their thresholds.

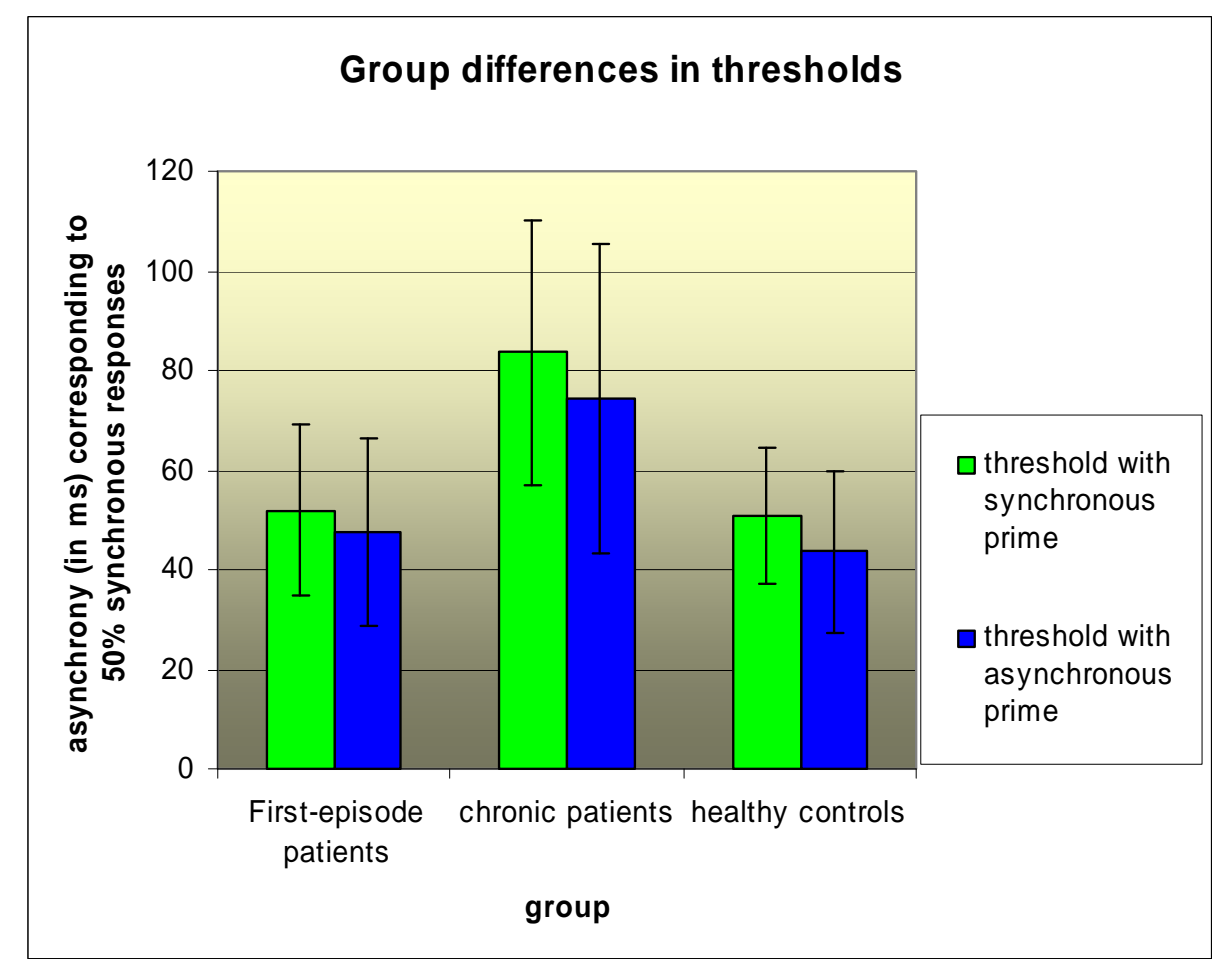

Figure 1: Group differences in visual simultaneity thresholds derived from the psychometric functions of FEP patients $(n=8)$, chronic patients $(n=6)$ and controls $(n=12)$. The standard deviations are also displayed for the various groups and tresholds. 
At closer inspection, particularly the chronic patient group could be divided into patients for which a psychometric function could be obtained and ones for which a psychometric functions could not be derived. These patients can be broadly divided into 3 groups. The first group appeared to be heavily influenced by the premask, this particular group of patients never showed a normal psychometric function but either stayed above or below threshold. A possible explanation for this behaviour could be that even though these patients knew that there should be successive and simultaneous conditions, they might not have seen simultaneous trials but were implicitly influenced by the effects of the premask. This would indicate that these patients do not have a perceptual problem but used other criteria to judge simultaneity. A second group of patients show a similar pattern to group 1 but after a certain SOA approach a threshold in the asynchronous premask conditions. Neuronal activation triggered by the asynchronous premask could account for this pattern as this is known to wear off after about 80 milliseconds. If patients used the absence of neuronal activity as a criterion to judge whether or not the two stimuli appeared simultaneously or successively they would be prone to respond synchronous unless their system had information available to contradict this assumption. The third group of patients never reached the $50 \%$ mark but either stayed above or below the $50 \%$ for both synchronous and asynchronous primes. These particular patterns of not following a normal psychometric function indicate that these patients do not experience a perceptual problem. It seems the case that these patients establish different criteria for judging simultaneity that are only partially successful. Two ways to explore this possibility exist. One solution would be to increase SOAs. It could be simply the case that some chronic schizophrenic patients have much higher thresholds than controls, in line with previous findings by Giersch et al. (2008).

In conclusion, the results from experiment 1 indicate that chronic patients appear to have higher thresholds as compared to healthy controls. FEP patients appear to have similar thresholds as control subjects. However, similar to the chronic sample, they show substantial variability, which might be explained by the different clinical presentations of the FEP sample. With regards to the chronic sample, substantial variability exists also in this sample, which could be related to particular subclasses of symptoms and will be explored in further analyses.

\section{Experiment 2:}

The results from this experiment are in line with experiment 1. A one-way ANOVA revealed significant group differences $\left(F_{2,50}=11.89\right.$, $\left.\mathrm{p}<0.01\right)$, which were accounted for by significant differences in thresholds between chronic patients (91 milliseconds) as compared to FEP patients (50 milliseconds) and healthy controls (46 milliseconds). Again, substantial variability existed in both the chronic and FEP patients' samples (see figure 2).

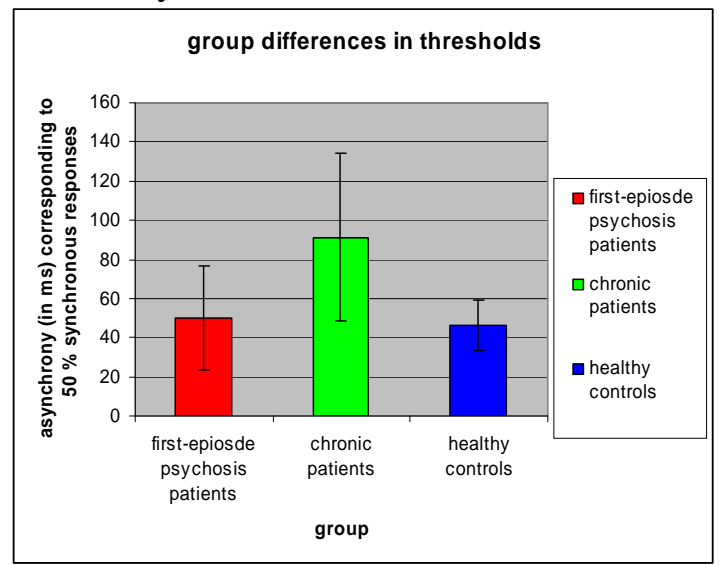

Figure 2: Group differences in visual simultaneity thresholds. The standard deviations are also displayed for the different groups. 
The results from experiment 1 confirm that chronic patients had substantially higher thresholds as compared to the FEP patients and healthy controls, even though both patient groups showed large variations within. Experiment 1 and experiment 2 showed a high correlation $(r=.85, p<0.01)$ indicating that both experiments use comparable measures of visual simultaneity thresholds.

The results from experiments 1 and 2 are in line with previous findings (e.g. Elliott et al., 2006, Giersch et al., 2008). Brecher (1932) originally reported thresholds of 55.3 milliseconds in healthy controls, similar to the 46 and 47 milliseconds found in this study. Similar to Giersch et al. (2008), chronic schizophrenic patients were found to have higher thresholds compared to controls. FEP patients showed similar thresholds as controls, however, there was substantial variability in their thresholds suggesting that subgroups of FEP patients might actually experience difficulties in event-structure coding, similar to chronic schizophrenic patients. The fact that some patients did not produce results in line with a normal psychometric function indicates that they might have problems choosing appropriate criteria for judging simultaneity. This will be explored by increasing the range of SOAs in case their thresholds are higher than 110 milliseconds.

\section{Acknowledgements}

This research is sponsored by a grant from Astra Zeneca.

\section{References}

Andreasen, N.C. (1999). A Unitary Model of Schizophrenia, Bleuler's "Fragmented Phrene” as Schizencephaly. Archives of General Psychiatry, 56, 781-787.

Brecher, G. A. (1932). Die Entstehung und biologische Bedeutung der subjectktiven Zeiteinheit - des Momentes. Zeitschrift für vergleichende Physiologie, 18, 204-243.

Davalos, D.B., Kisley, M.A. \& Ross, R. G. (2002). Deficits in auditory and visual temporal perception in schizophrenia. Cognitive Neuropsychiatry, 7 (4), 273-282.

Elliott, M. A., Shi, Z. \& Sürer, F. (2006). The effects of subthreshold synchrony on the perception of simultaneity. Psychological Research,

Giersch, A., Lalanne, L., Corves, C., Seubert, J., Shi, Z., Foucher, J. and Elliott, M.A. (2008). Extended Visual Simultaneity Tresholds in Patients with Schizophrenia.

Schizophrenia Bulletin, March 21

Treutwein,B. (1995). Adaptive psychophysical procedures. Vision Research, 35, 2503-2522. 